\title{
A New (Cartesian) Reaction-path Model for Dynamics in Polyatomic Systems, with Application to $\mathrm{H}$-atom Transfer in Malonaldehyde
}

\author{
Beverly A. Ruf and William H. Miller* \\ Department of Chemistry, University of California, and Material and Chemical Sciences \\ Division, Lawrence Berkeley Laboratory, Berkeley, California 94720, U.S.A.
}

\begin{abstract}
A new kind of reaction-path model for describing reactions in polyatomic molecular systems is presented, based on the cartesian coordinates of the atoms. Not only does this lead to a simpler treatment of the interaction between the 'system' (i.e. the reaction coordinate) and the 'bath' (the other degrees of freedom) than earlier reaction-path models based on the (curvilinear) steepest-descent reaction path, but in many cases it also provides a more natural description of the dynamics. The resulting Hamiltonian has the standard form of a cartesian 'system' linearly coupled to a harmonic 'bath', the dynamics of which is treated in this paper by the basis set method of Makri and Miller (J. Chem. Phys., 1987, 86, 1451). Application to a model of $\mathrm{H}$-atom transfer in malonaldehyde shows that the overall approach (both the cartesian reaction-path model and the basis-set method treatment of the dynamics) is quite successful.
\end{abstract}

\section{Introduction}

Over the past seven years or so our research group has developed (and applied) a theoretical description of dynamics in polyatomic molecular systems that is based on the minimum-energy reaction path (i.e. the 'intrinsic reaction coordinate'). ${ }^{1-3}$ This is the steepest-descent path (in mass-weighted cartesian coordinates) from the transition state on a potential-energy surface backwards to reactants and forward to products. The picture of the dynamics in this model is that of one-dimensional motion along the reaction path coupled to harmonic vibration about it (in $3 N-7$ directions, where $N$ is the number of atoms). Although this model has been useful for a variety of applications, and we believe will continue to be so, there are some aspects of it that are undesirable. The purpose of this paper is to present a new class of models for describing dynamics in polyatomic systems that seems more advantageous in some respects than that based on the minimum-energy reaction path.

One type of process for which the reaction-path has difficulty is hydrogen-atom transfer as, for example, in malonaldehyde, ${ }^{\prime \prime}$<smiles>[Y]C([Y])C</smiles> 
The reactive process is clearly the motion of essentially only one hydrogen atom, $\mathrm{H}_{1}$, with the other degrees of freedom playing a modest role. The problem with the reaction-path description here is that the reaction path always arrives at the reactant and product wells along the normal mode of lowest frequency (of the appropriate symmetry); $c f$. Appendix 1. For the above example (1) the lowest frequency is associated with some floppy skeletal vibrational motion that is quite unrelated to the motion of atom $\mathrm{H}_{1}$, while the relevant vibration is the $\mathrm{O}-\mathrm{H}_{1}$ stretch, which is the highest frequency of the reactant. This means that in going from the transition state to the reactant and product wells the minimum-energy path makes many sharp turns in the $(3 N-6)$ dimensional space, so that the reaction is in no sensible way well described as being one-dimensional motion along this path. (The curvature coupling elements in the reaction path Hamiltonian ${ }^{1 a}$ are correspondingly large and difficult to deal with.)

Another drawback of the reaction-path model is that the reaction path is massdependent. Thus, if one wishes to treat isotopically related reactions, an entire recalculation of the minimum-energy path (and force constant matrix along it) is required for each new isotopic species. One would clearly prefer a model for which the potentialenergy surface 'input' to the dynamical treatment is independent of the nuclear masses.

The new model that we describe in section 2 is from the outset much simpler than our earlier reaction-path models. Cartesian coordinates of the $N$ atoms are used for all degrees of freedom, and one relies on 'chemical intuition' to select which one (or ones) is treated for arbitrarily large displacements, as opposed to the other coordinates which move only slightly. For reaction (1), for example, one notes that it is only the $x$ coordinate of hydrogen atom $\mathrm{H}_{1}$ that undergoes large displacement; although all the other atoms (and the $y$ and $z$ coordinate of $\mathrm{H}_{1}$ ) move, they do not move very much. Thus, the potential-energy surface in all these cartesian coordinates can be adequately approximated by a quadratic expansion about some reference configuration. Conservation of total angular momentum is accounted for approximately (but adequately for $\mathrm{H}$-atom transfer processes).

The Hamiltonian which characterizes this new approach is totally cartesian in structure and thus much simpler to deal with than the reaction-path Hamiltonian. In fact, it has the generic form of a 'system' which is linearly coupled to a harmonic 'bath', as has been so commonly assumed for many model studies. ${ }^{4}$ It is much easier to describe the coupling between the 'system' and 'bath' here than it is in the reaction-path Hamiltonian where the coupling arises from curvature coupling effects in the kinetic energy. ${ }^{1 a}$ Finally, the potential-energy surface information required for this cartesian model is simpler to generate than that for the reaction path Hamiltonian, and it is also mass-independent so that different isotopes of the same system do not require new potential-energy surface calculations.

The cartesian reaction-path model is developed in section 2, and section 3 describes the theoretical methods used to treat the dynamics. Application to a model of $\mathrm{H}$-atom transfer in malonaldehyde is presented in section 4 .

\section{A Cartesian Model for Reaction Dynamics}

\section{(a) Frozen Bath Version}

We start with the Hamiltonian for an $\mathrm{N}$-atom polyatomic system expressed in the full set of $3 N$ cartesian coordinates:

$$
H=\sum_{i=1}^{3 N} \frac{p_{i}^{2}}{2 m_{i}}+V\left(\left\{x_{i}\right\}\right)
$$

The first step in defining the model is to identify which cartesian coordinate(s) one wishes to describe for arbitrarily large displacements. For reaction (1) one could choose 
only the $x$ coordinate of hydrogen atom $\mathrm{H}_{1}$; a more accurate model would be to treat both the $x$ and $y$ coordinates of $\mathrm{H}_{1}$ for arbitrarily large displacement. Let $\boldsymbol{r}$ denote the one or two (or maybe three) cartesian coordinates to be described for arbitrary displacements; $\boldsymbol{R}$ denotes the remaining $3 N-1$ or $3 N-2$ cartesian coordinates that do not move very much during the reaction of interest. The $\boldsymbol{R}$-dependence of the potential energy, $V=V(\boldsymbol{r}, \boldsymbol{R})$ is then expanded in a Taylor series to second order about a reference geometry $\boldsymbol{R}_{0}$ :

$$
\begin{aligned}
V(\boldsymbol{r}, \boldsymbol{R})= & V\left(\boldsymbol{r}, \boldsymbol{R}_{0}\right)+\frac{\partial V\left(\boldsymbol{r}, \boldsymbol{R}_{0}\right)}{\partial \boldsymbol{R}_{0}} \cdot\left(\boldsymbol{R}-\boldsymbol{R}_{0}\right) \\
& +\frac{1}{2}\left(\boldsymbol{R}-\boldsymbol{R}_{0}\right) \cdot \frac{\partial^{2} V\left(\boldsymbol{r}, \boldsymbol{R}_{0}\right)}{\partial \boldsymbol{R}_{0} \partial \boldsymbol{R}_{0}} \cdot\left(\boldsymbol{R}-\boldsymbol{R}_{0}\right) .
\end{aligned}
$$

Eqn (3) is the essence of the model, exploiting the fact that most of the coordinates (i.e. atoms) do not move very much during the reaction.

With eqn (3) the Hamiltonian takes the form

$$
\begin{aligned}
H(\boldsymbol{p}, \boldsymbol{r}, \boldsymbol{P}, \boldsymbol{R})= & {\left[\frac{1}{2} \boldsymbol{p} \cdot \mathbf{m}^{-1} \cdot \boldsymbol{p}+V_{0}(\boldsymbol{r})\right] } \\
& +\left[\frac{1}{2} \boldsymbol{P} \cdot \mathbf{m}^{-1} \cdot \boldsymbol{P}+\frac{1}{2}\left(\boldsymbol{R}-\boldsymbol{R}_{0}\right) \cdot \mathbf{K}(\boldsymbol{r}) \cdot\left(\boldsymbol{R}-\boldsymbol{R}_{0}\right)\right]-\boldsymbol{f}(\boldsymbol{r}) \cdot\left(\boldsymbol{R}-\boldsymbol{R}_{0}\right)
\end{aligned}
$$

which one recognizes as essentially the generic Hamiltonian for a (low-dimensional) 'system' [the $\boldsymbol{r}$ degree(s) of freedom] coupled to a 'bath' of harmonic oscillators, the $\boldsymbol{R}$ degrees of freedom. [The quantities in eqn (4) are clearly recognizable: $V_{0}(r)=$ $\boldsymbol{V}\left(\boldsymbol{r}, \boldsymbol{R}_{0}\right), \mathbf{K}(\boldsymbol{r})=\partial^{2} \boldsymbol{V}\left(\boldsymbol{r}, \boldsymbol{R}_{0}\right) / \partial \boldsymbol{R}_{0} \partial \boldsymbol{R}_{0}, \boldsymbol{f}(\boldsymbol{r})=-\partial \boldsymbol{V}\left(\boldsymbol{r}, \boldsymbol{R}_{0}\right) / \partial \boldsymbol{R}_{0}$ and $\mathbf{m}$ is the diagonal matrix of atomic masses.] The coupling [the last term in eqn (4)] is linear in the 'bath' coordinates $\boldsymbol{R}$. This describes 'solvent-reorganization' effects, i.e. the change in the 'bath' coordinates' instantaneous equilibrium positions as the 'system' dynamics takes place. There is also another coupling between the 'system' and 'bath' because the force constant matrix of the bath is a function of the 'system' coordinate $r$; i.e. the instantaneous vibrational frequencies and normal-mode eigenvectors change with the system dynamics. One familiar with the reaction-path formalism will immediately recognize how much more readily one can deal with system-bath coupling with this new Hamiltonian, eqn (4), than with that in the reaction-path Hamiltonian. ${ }^{1 a}$

The reader will also note that the required input from $a b$ initio quantum chemistry calculations is much easier to generate for the Hamiltonian in eqn (4) than it is for the reaction-path Hamiltonian. What is required for eqn (4) is the energy, gradient and force constant matrix of the potential-energy surface for a frozen bath $\left(\boldsymbol{R}=\boldsymbol{R}_{0}\right)$, as a function of $\boldsymbol{r}$. If $\boldsymbol{r}$ is only one coordinate, for example, this means that one requires an energy, gradient and force constant matrix at, say, ten predetermined geometries. Geometry optimization is not required.

\section{(b) Conservation of Total Angular (and Linear) Momentum}

Our initial inclination was to ignore the fact that this model does not conserve total angular and linear momentum (owing to the fact that the approximation to the potential surface, eqn (3), destroys rotational and translational invariance). Earlier, for example, Jaquet and Miller ${ }^{5}$ had used essentially this model to treat $\mathrm{H}$-atom diffusion on a tungsten surface, and there, of course, one does not need to be concerned with rotation and translation of the (infinite) surface of tungsten atoms. In malonaldehyde [eqn (1.)], however, the 'substrate' is not infinite. It seemed to us that it was sufficiently more massive than the one $\mathrm{H}$ atom that tunnels and that one could thus ignore rotation and translation. Unfortunately, test calculations convinced us that this is not the case, at 
least for malonaldehyde; the low frequencies of the bath (those that should be zero, i.e. pure rotations and translations) mix in an unphysical way with the true low vibrational frequencies of the molecule. It was thus deemed necessary to project out six pure rotational and translational degrees of freedom.

To separate off the rotational motion rigorously requires use of curvilinear coordinates (i.e. Euler angles), ${ }^{6}$ thus destroying the cartesian form of the Hamiltonian [eqn (4)]. To avoid this, and also because we are developing the present model for application to large molecular systems for which rotational motion is not of interest, we have chosen to eliminate rotation approximately by projecting out infinitesimal rotation and translation of the $N-1$ 'substrate' atoms.

To be specific, we refer to eqn (1) with $\boldsymbol{r}=x_{\mathrm{H}_{1}}$ chosen as the (one) 'system' coordinate. Indices $i=1-3$ refer to the three cartesian coordinates of atom $H_{1}$. The matrix

$$
\begin{aligned}
\left\{\mathbf{U}_{i, k}\right\} & i=4, \ldots, 3 N \\
k & =4, \ldots, 3 N-6
\end{aligned}
$$

denotes the matrix of eigenvectors of the projected, mass-weighted force constant matrix of the $N-1$ atom substrate,

$$
(1-\mathbf{P}) \cdot \mathbf{m}^{-1 / 2} \cdot \mathbf{K}_{0} \cdot \mathbf{m}^{-1 / 2} \cdot(1-\mathbf{P})
$$

Here $\mathbf{K}_{0} \equiv \mathbf{K}\left(x_{1}^{0}\right), \mathbf{m}$ and $\mathbf{P}$ are $(3 N-3) \times(3 N-3)$ matrices for the $(N-1)$-atom substrate, and $x_{1}^{0}$ is some convenient intermediate value of the system coordinate $x_{1}$. $\mathbf{P}$ is the projector onto the 6 degrees of freedom that are infinitesimal rotations and translations of the $(N-1)$ atom substrate; its explicit form has been given before ${ }^{1 a}$ and is a function only of the geometry of the substrate. The factor $(1-\mathbf{P})$ in eqn (6) insures that the projected, mass-weighted force constant matrix will have six zero eigenvalues, corresponding to infinitesimal rotations and translations, and $3(N-1)-6$ non-zero eigenvalues that describe vibration of the $(N-1)$ substrate atoms about their reference positions.

The matrix $U$ is now augmented by the other cartesian coordinates of atom $\mathrm{H}_{1}$ that are not taken as system coordinates; e.g. with $r$ chosen to be $x_{\mathrm{H}_{1}}=x_{1}$, the coordinates $y_{\mathbf{H}_{1}}=x_{2}$ and $z_{\mathbf{H}_{1}}=x_{3}$ are part of the bath, and the final $\mathbf{U}$ matrix is

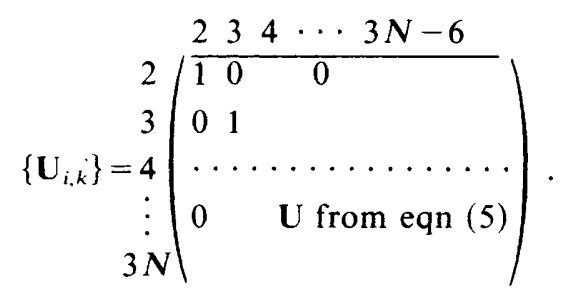

The $(3 N-1)$ cartesian bath coordinates $R$ are now expressed in terms of the $3 N-7$ normal mode bath coordinates $\boldsymbol{Q}$ which have the six rotations and translations of the substrate eliminated,

$$
\boldsymbol{R}-\boldsymbol{R}_{0}=\mathbf{m}^{-1 / 2} \cdot \mathbf{U} \cdot \boldsymbol{Q}
$$

with $\mathbf{U}$ given by eqn (7). The Hamiltonian of eqn (4) thus becomes (with $r \equiv x_{1}$ )

$$
\begin{aligned}
H\left(p_{1}, x_{1}, \boldsymbol{P}, \boldsymbol{Q}\right)= & \left(\frac{p_{1}^{2}}{2 m_{1}}+V_{\mathrm{eff}}\left(x_{1}\right)\right) \\
& +\left(\sum_{k=2}^{3 N-6} \frac{1}{2} P_{k}^{2}+\frac{1}{2} \boldsymbol{Q} \cdot \mathbf{K}_{\mathrm{eff}}\left(x_{1}\right) \cdot \boldsymbol{Q}\right)-\boldsymbol{f}_{\mathrm{eff}}\left(x_{1}\right) \cdot \boldsymbol{Q}
\end{aligned}
$$


where $\mathbf{K}_{\mathrm{eff}}$ and $\boldsymbol{f}_{\mathrm{eff}}$ are the $(3 N-7)$ matrix and vector, respectively,

$$
\begin{aligned}
\mathbf{K}_{\mathrm{eff}}\left(x_{1}\right) & =\mathbf{U}^{+} \cdot \mathbf{m}^{-1 / 2} \cdot \boldsymbol{K}\left(x_{1}\right) \cdot \mathbf{m}^{-1 / 2} \cdot \mathbf{U} \\
\boldsymbol{f}_{\mathrm{eff}}\left(x_{1}\right) & =\boldsymbol{f}\left(x_{1}\right) \cdot \mathbf{m}^{-1 / 2} \cdot \mathbf{U}
\end{aligned}
$$

and

$$
V_{\text {eff }}\left(x_{1}\right)=V\left(x_{1}, \boldsymbol{R}_{0}\right) \text {. }
$$

[Note that $\mathbf{K}_{\mathrm{eff}}\left(x_{1}\right)$ is not diagonal because $\mathbf{U}$ was obtained by diagonalizing $\mathbf{K}\left(x_{1}\right)$ at the fixed value $x_{1}=x_{1}^{0}$.] This Hamiltonian, eqn (9), is one for $3 N-6$ degrees of freedom. It should be clear how eqn (5)-(9) are modified if the system coordinate(s) $\boldsymbol{r}$ is chosen to be $x_{1}$ and $x_{2} \equiv y_{\mathrm{H}_{1}}$, say.

The transformations described by eqn (5)-(9) for eliminating overall translation and rotation of the substrate must be re-done for different isotopic species; i.e. the matrix $\mathbf{K}_{\text {eff }}\left(x_{1}\right)$ and coupling vector $f_{\text {eff }}\left(x_{1}\right)$ in eqn (9) depend on the atomic masses. It should be noted, however, that the mass dependence on the projected $(3 N-3) \times(3 N-3)$ substrate portion of $\mathbf{K}_{\text {eff }}\left(x_{1}\right)$ corresponds to secondary isotope effects. Primary isotopic substitutions are simply incorporated via the identity portion of the matrix $\mathbf{U}$. Both of these mass variations are simple, though. The important matter is that the original $\mathbf{K}$ and $f$ of eqn (4), which are obtained from ab initio quantum-chemistry calculations, are mass-independent. Also important for treating the dynamics of the 'system-bath' Hamiltonian [eqn (9)] is that its cartesian and linear coupling form has been maintained by the (approximate) way that we have eliminated overall translation and rotation.

\section{(c) Flexible Bath}

In some cases one may wish to allow the reference geometry of the 'bath' to vary with the system coordinate $r$, e.g. so that the equilibrium geometries of the reactants and products are accurately reproduced by the model. It is actually possible to generalize the above treatment in a simple way to incorporate this and still maintain the simple form of the resulting Hamiltonian.

Thus, let $\boldsymbol{R}_{0}(\boldsymbol{r})$ be the reference geometry of the bath as a function of the system coordinate(s) $\boldsymbol{r}$. We envision, for example, that $\boldsymbol{R}_{0}(\boldsymbol{r})$ may be chosen simply to interpolate between the reactant and product geometries of the bath variables. A Taylor-series expansion of $\boldsymbol{R}$ about $\boldsymbol{R}_{0}(\boldsymbol{r})$, as in eqn (3), is still possible:

$$
\begin{aligned}
V(\boldsymbol{r}, \boldsymbol{R})= & V\left[\boldsymbol{r}, \boldsymbol{R}_{0}(\boldsymbol{r})\right]+\left(\frac{\partial V(\boldsymbol{r}, \boldsymbol{R})}{\partial \boldsymbol{R}}\right)_{\boldsymbol{R}=\boldsymbol{R}_{0}(\boldsymbol{r})} \cdot\left[\boldsymbol{R}-\boldsymbol{R}_{0}(\boldsymbol{r})\right] \\
& +\frac{1}{2}\left[\boldsymbol{R}-\boldsymbol{R}_{0}(\boldsymbol{r})\right] \cdot\left(\frac{\partial^{2} V(\boldsymbol{r}, \boldsymbol{R})}{\partial \boldsymbol{R} \partial \boldsymbol{R}}\right)_{\boldsymbol{R}=\boldsymbol{R}_{0}(\boldsymbol{r})} \cdot\left[\boldsymbol{R}-\boldsymbol{R}_{0}(\boldsymbol{r})\right]
\end{aligned}
$$

and this can be combined with the cartesian kinetic energy to form a Hamiltonian like that of eqn (4).

To maintain the simple form of the resulting Hamiltonian it is still necessary to project out rotations and translations of a frozen substrate. Therefore, the transformation matrix $\mathbf{U}$ is defined as above [eqn (5)-(7)] but where $\mathbf{K}_{0}=\mathbf{K}\left[x_{1}^{0}, \boldsymbol{R}_{0}\left(x_{1}^{0}\right)\right], x_{1}^{0}$ being an intermediate value of the system coordinate $x_{1}$. The projector $\mathbf{P}$ is also defined at the frozen substrate geometry $\boldsymbol{R}_{0}\left(x_{1}^{0}\right)$. The relation between the $(3 N-1)$ coordinates $\boldsymbol{R}$ (for $\left.\boldsymbol{r} \equiv x_{1}\right)$ and the $(3 N-7)$ coordinates $Q$ is thus

$$
\boldsymbol{R}-\boldsymbol{R}_{0}\left(x_{1}^{0}\right)=\mathbf{m}^{-1 / 2} \cdot \mathbf{U} \cdot \boldsymbol{Q}
$$

so that $\boldsymbol{R}-\boldsymbol{R}_{0}\left(x_{1}\right)$ in eqn (10) (with $\boldsymbol{r} \equiv x_{1}$ ) is given by

$$
\boldsymbol{R}-\boldsymbol{R}_{0}\left(x_{1}\right)=\boldsymbol{R}_{0}\left(x_{1}^{0}\right)-\boldsymbol{R}_{0}\left(x_{1}\right)+\mathbf{m}^{-1 / 2} \cdot \mathbf{U} \cdot \boldsymbol{Q} .
$$


Using eqn (12) in eqn (10) then gives the same form of Hamiltonian as before [eqn $(9 a)]$ here with

$$
\begin{gathered}
\mathbf{K}_{\mathrm{eff}}\left(x_{1}\right)=\mathbf{U}^{+} \cdot \mathbf{m}^{-1 / 2} \cdot \mathbf{K}\left(x_{1}\right) \cdot \mathbf{m}^{-1 / 2} \cdot \mathbf{U} \\
\boldsymbol{f}_{\mathrm{eff}}\left(x_{1}\right)= \\
V_{\mathrm{eff}}\left(x_{1}\right)= \\
\left.\left.\boldsymbol{f}\left(x_{1}\right)+\left[\boldsymbol{R}_{0}\left(x_{1}\right)-\boldsymbol{R}_{0}\left(x_{1}^{0}\right)\right] \cdot \mathbf{K}\left(x_{1}\right)\right\} \cdot \mathbf{m}^{-1 / 2}\left(x_{1}\right)\right]+\boldsymbol{U}\left(x_{1}\right) \cdot\left[\boldsymbol{R}_{0}\left(x_{1}\right)-\boldsymbol{R}_{0}\left(x_{1}^{0}\right)\right] \\
+\frac{1}{2}\left[\boldsymbol{R}_{0}\left(x_{1}\right)-\boldsymbol{R}_{0}\left(x_{1}^{0}\right)\right] \cdot \mathbf{K}\left(x_{1}\right) \cdot\left[\boldsymbol{R}_{0}\left(x_{1}\right)-\boldsymbol{R}_{0}\left(x_{1}^{0}\right)\right]
\end{gathered}
$$

where

$$
\begin{aligned}
& \mathbf{K}\left(x_{1}\right)=\left(\frac{\partial^{2} V\left(x_{1}, \boldsymbol{R}\right)}{\partial \boldsymbol{R} \partial \boldsymbol{R}}\right)_{\boldsymbol{R}=\boldsymbol{R}_{0}\left(x_{1}\right)} \\
& \boldsymbol{f}\left(x_{1}\right)=-\left(\frac{\partial V\left(x_{1}, \boldsymbol{R}\right)}{\partial \boldsymbol{R}}\right)_{\boldsymbol{R}=\boldsymbol{R}_{0}\left(x_{1}\right)} .
\end{aligned}
$$

It is clear that these expressions revert to eqn (9) in the rigid bath limit, where $\boldsymbol{R}_{0}\left(x_{1}\right)=\boldsymbol{R}_{0}$.

To conclude this description of the model we summarize the advantages that it has over earlier reaction-path models. (1) Most important is that it contains the physically correct picture of the dynamics, clearly identifying the relevant coordinates of the process. The intrinsic reaction path for these heavy-light-heavy mass combinations leads to unphysical reaction paths. ${ }^{7}$ (2) It is considerably easier to treat the dynamics of the resulting Hamiltonian which has the coupling in the potential energy rather than that for the reaction path Hamiltonian that has the coupling in the kinetic energy. (3) It is relatively simple to do calculations for different isotopes. (4) It is much simpler to generate the $a b$ initio quantum chemistry 'input' for the model.

Disadvantages of the model, on the other hand, are that we are able to separate-off overall rotational motion only approximately if we wish (as we do) to maintain the simple form of the Hamiltonian; this seems to be a minor error, particularly so if the 'substrate' is large. Finally, the model requires that one invoke 'chemical intuition' to choose the cartesian coordinates that constitute the 'system'; one may view this as an advantage or a disadvantage.

\section{System-Bath Dynamics}

Having defined the model Hamiltonian in the previous section, one is now ready to treat its dynamics. The most elegant and rigorous way to treat these system-harmonic bath Hamiltonians is via Feynman path integral methods. ${ }^{8}$ This permits one to take into account the effect of the 'bath' on the 'system' exactly. There is currently a great deal of progress being made in this direction, ${ }^{5.9-14}$ but these approaches are at present not available for practical calculations with real chemical systems. In this paper, therefore, we utilize an approximate treatment due to Makri and $\mathrm{Miller}^{4}$ that has been shown to do an excellent job for including the effect of coupling to a bath on the tunnelling in a double-well system, as is the process in eqn (1). We first summarize the basic ideas of this approach and then describe some necessary modifications for the present application.

The basis-set method of Makri and Miller chooses basis functions for the total system-bath Hamiltonian in the form

$$
\psi_{i n}(r, \boldsymbol{Q})=\chi_{i}(r) \Phi_{n}^{i}(\boldsymbol{Q})
$$

where $\left\{\chi_{i}(r)\right\}$ is a set of localized functions in the 'system' coordinate and $\Phi_{n}^{\prime}(Q)$ is the eigenfunction of the 'bath' Hamiltonian that results when the total Hamiltonian is averaged over basis function $\chi_{i}(r) ; \Phi_{n}^{i}(Q)$ is obtainable analytically because the resulting 
'bath' Hamiltonian is that of linearly coupled harmonic oscillators. (We have taken the set $\left\{\chi_{i}\right\}$ as the distributed Gaussians of Hamilton and Light, ${ }^{15}$ as used by Makri and Miller.) The matrix of the total system-bath Hamiltonian, $H_{i^{\prime} n^{\prime}, \text { in }}$, is constructed in this basis, and the zeroth-order effective system Hamiltonian of ref. (4) is defined by taking the part of the Hamiltonian matrix that is diagonal in the 'bath' quantum numbers, i.e. by setting $\boldsymbol{n}^{\prime}=\boldsymbol{n}$. This effective system Hamiltonian matrix has a dimension only of the number of 'system' basis functions $\left\{\chi_{i}\right\}$ and is of the form

$$
\begin{aligned}
H_{i^{\prime}, i}^{n} & \equiv H_{i^{\prime}, \text { in }} \\
& =F_{i^{\prime}, i}^{n} h_{i^{\prime}, i}^{n}
\end{aligned}
$$

where $F_{i^{\prime}, i}^{n}$ is the Franck-Condon factor between the oscillator functions $\Phi_{n}^{i}$ and $\Phi_{n}^{i^{\prime}}$,

$$
F_{i^{\prime}, i}^{n}=\int \Phi_{n}^{i^{\prime}}(\boldsymbol{Q}) \Phi_{n}^{i}(\boldsymbol{Q}) \mathrm{d} \boldsymbol{Q}
$$

and $h_{i, i}^{n}$ is a one-dimensional-like Hamiltonian matrix. Most of the effects of the 'bath' on the 'system' dynamics are contained in the Franck-Condon factor; i.e. it describes the 'solvent relaxation', or polaronic effects of the 'bath' on the 'system'. Makri and Miller found that this approximation, i.e. taking the bath quantum numbers to be diagonal, worked quite well provided that the basic functions $\left\{\chi_{i}\right\}$ are localized. The reader should see ref. (4) for discussion of the reasons for this as well as further aspects of the approach.

Makri and Miller considered a system-bath Hamiltonian with a constant force constant matrix, $\mathbf{K}$, so their treatment must be generalized in order to apply it to the present Hamiltonian [eqn (9a)]. Specifically, the Franck-Condon factor of eqn (15) in the present case is given more explicitly by

$$
F_{i^{\prime}, i}^{n}=\int \prod_{k} \phi_{n_{k}}\left(q_{k}^{i^{\prime}}\right) \phi_{n_{k}}\left(q_{k}^{i}\right) \mathrm{d} \boldsymbol{Q}
$$

where $\phi_{n_{k}}\left(q_{k}\right)$ are ordinary one-dimensional harmonic oscillator wavefunctions, and the coordinates $\boldsymbol{q}^{i}$ (and $\boldsymbol{q}^{i^{\prime}}$ ) are the linear combinations of $\boldsymbol{Q}$ that diagonalize the oscillator potentials

$$
\frac{1}{2} \boldsymbol{Q} \cdot \mathbf{K}_{i} \cdot \boldsymbol{Q}-\boldsymbol{f}_{i} \cdot \boldsymbol{Q}
$$

where

$$
\begin{aligned}
\mathbf{K}_{i} & =\left\langle\chi_{i}\left|\mathbf{K}_{\mathrm{eff}}\right| \chi_{i}\right\rangle \\
\boldsymbol{f}_{i} & =\left\langle\chi_{i}\left|\boldsymbol{f}_{\mathrm{eff}}\right| \chi_{i}\right\rangle .
\end{aligned}
$$

More specifically, if $\mathbf{L}_{i}$ is the $(3 N-7) \times(3 N-7)$ matrix of eigenvectors of $\mathbf{K}_{i}$, then

$$
\mathbf{L}_{i}^{+} \cdot \mathbf{K}_{i} \cdot \mathbf{L}_{i}=\left(\boldsymbol{\omega}_{i}\right)^{2} \mathbf{1}
$$

are the diagonal frequencies of the bath modes associated with the Gaussian $\chi_{i}$ localized at $\boldsymbol{r}_{i}$, and $\boldsymbol{q}^{i}$ is given in terms of $\boldsymbol{Q}$ by

$$
\boldsymbol{q}^{i}=\mathbf{L}_{i}^{+} \cdot \boldsymbol{Q}-\boldsymbol{\omega}_{i}^{-2} \cdot \mathbf{L}_{i}^{+} \cdot \boldsymbol{f}_{i}
$$

Because the matrices $\mathbf{L}_{i}$ and $\mathbf{L}_{i}$ are different (owing to the fact that the force constant matrices $\mathbf{K}_{i}$ and $\mathbf{K}_{i^{\prime}}$ are different) the integrals in eqn (16) do not factorize into a product of one-dimensional integrals; they have the form of a multidimensional Gaussian integral (times powers). There have, however, been a number of papers describing the efficient evaluation of these multidimensional harmonic Franck-Condon factors using generating function, recursion and iterative methods ${ }^{16}$ we used a method similar to that of ref. $(16 a)$. 
With this more generalized Franck-Condon factor, the effective system Hamiltonian which results from the system-bath Hamiltonian of eqn $(9 a)$ is given explicitly by

$$
\begin{aligned}
H_{i^{\prime}, i}^{n}= & F_{i^{\prime}, i}^{n}\left\langle\chi_{i^{\prime}}\left|\frac{p_{1}^{2}}{2 m_{1}}+V_{\mathrm{eff}}\left(x_{1}\right)\right| \chi_{i}\right\rangle+\frac{1}{2}\left\langle\chi_{i^{\prime}} \mid \chi_{i}\right\rangle\left\langle\Phi_{n}^{i^{\prime}}\left|\boldsymbol{P}^{2}\right| \Phi_{n}^{i}\right\rangle \\
& +\frac{1}{2}\left\langle\Phi_{n}^{i}\left|\boldsymbol{Q} \cdot \mathbf{K}_{i^{\prime} i} \cdot \boldsymbol{Q}\right| \Phi_{n}^{i}\right\rangle-\boldsymbol{f}_{i^{\prime} i} \cdot\left\langle\Phi_{n}^{i}|\boldsymbol{Q}| \Phi_{n}^{i}\right\rangle
\end{aligned}
$$

where

$$
\begin{aligned}
\mathbf{K}_{i^{\prime} i} & =\left\langle\chi_{i^{\prime}}\left|\mathbf{K}_{\mathrm{eff}}\left(x_{1}\right)\right| \chi_{i}\right\rangle \\
\boldsymbol{f}_{i^{\prime} i} & =\left\langle\chi_{i^{\prime}}\left|\boldsymbol{f}_{\mathrm{eff}}\left(x_{1}\right)\right| \chi_{i}\right\rangle .
\end{aligned}
$$

The last three terms in eqn (20) are multidimensional Gaussians times powers and are evaluated by methods similar to those used for the Franck-Condon factor.

\section{Application to Three-atom Model of Malonaldehyde}

Before applying the methodology of sections 2 and 3 to an $a b$ initio treatment of malonaldehyde [eqn (1)] we consider here a simple three-atom model of this reaction that can be treated exactly (because it is a triatomic system). It is important to test both the cartesian reaction-path model described in section 2 and also the dynamical treatment summarized in section 3 .

The three-atom model we consider is the $\mathrm{O}-\mathrm{H}-\mathrm{O}$ part of eqn (1),<smiles>O[14CH2]O[14CH2]O[14CH2]O</smiles>

with a potential-energy function that has the general form of the double-well potential of Janoschek et al., ${ }^{17}$ generalized to include bending motion:

$$
V=a_{1}+a_{2} Q_{\mathrm{a}}^{2}+a_{3} Q_{\mathrm{a}}^{4}+a_{4} Q_{\mathrm{s}}^{2}+a_{5} Q_{\mathrm{a}}^{2} Q_{\mathrm{s}}+a_{6} Q_{\mathrm{s}}^{2}+\frac{1}{2} K_{\gamma}\left(\gamma-\gamma_{0}\right)^{2}
$$

where $\gamma$ is the angle between the two $\mathrm{OH}$ bonds. The coefficients (in a.u.) have been chosen to approximate the energetics and geometry of reaction (1):

$$
\begin{aligned}
a_{1} & =0.01338 \\
a_{2} & =-0.03603 \\
a_{3} & =0.02425 \\
a_{4} & =-0.02745 \\
a_{5} & =0.03695 \\
a_{6} & =0.07786 \\
K_{\gamma} & =0.20000 \\
Q_{a} & =\sqrt{\frac{1}{2}}\left(r_{1}-r_{2}\right) \\
Q_{5} & =\sqrt{\frac{1}{2}}\left(r_{1}+r_{2}\right) \\
\gamma_{0} & =158^{\circ} .
\end{aligned}
$$

The 'bare' barrier height (i.e. with no zero-point energy corrections) for this potential is $6.88 \mathrm{kcal} \mathrm{mol}^{-1}$, in qualitative agreement with what is thought to be the correct value for malonaldehyde. ${ }^{18.19}$ Table 1 compares the equilibrium and transition-state geometries for this potential to the corresponding quantities for malonaldehyde. 
Table 1. Comparison of geometries of the three-atom model to malonaldehyde

\begin{tabular}{|c|c|c|c|c|}
\hline \multirow[b]{2}{*}{$\begin{array}{c}\text { internal } \\
\text { coordinates }\end{array}$} & \multicolumn{2}{|c|}{ equilibrium } & \multicolumn{2}{|c|}{ transition state } \\
\hline & $\begin{array}{l}\text { three-atom } \\
\text { model }\end{array}$ & malonaldehyde ${ }^{h}$ & $\begin{array}{l}\text { three-atom } \\
\text { model }\end{array}$ & malonaldehyde ${ }^{h}$ \\
\hline$r_{1} / \AA$ & 0.82 & 0.99 & 1.20 & 1.20 \\
\hline$r_{2} / \AA$ & 1.46 & 1.69 & 1.20 & 1.20 \\
\hline$r_{3} / \AA$ & 2.23 & 1.22 & 2.36 & 2.36 \\
\hline$\gamma /^{\circ}$ & 158 & 155 & 158 & 158 \\
\hline
\end{tabular}

${ }^{a} r_{1}$ and $r_{2}$ and $\mathrm{OH}$ bond lengths; $r_{3}$ is the $\mathrm{O}-\mathrm{O}$ bond length; $\gamma$ is the angle between the two $\mathrm{OH}$ bonds. "See ref. (18) and (19).

Table 2. Tunnelling splitting $\left(\mathrm{cm}^{-1}\right)$ for the three-atom model of malonaldehyde

\begin{tabular}{|c|c|c|c|c|}
\hline \multirow[b]{2}{*}{ isotope } & \multicolumn{4}{|c|}{ reference geometry of bath } \\
\hline & transition state ${ }^{a}$ & equilibrium $^{a}$ & flexible ${ }^{h}$ & exact $^{c}$ \\
\hline $\mathrm{OHO}$ & $81(156)^{d}$ & $76(63)$ & $66(95)$ & 76 \\
\hline ODO & $23(30)$ & $9(18)$ & $7(22)$ & 9 \\
\hline
\end{tabular}

The cartesian reaction model of section 2 was now applied, with the $x$-coordinate of the $\mathrm{H}$-atom as the 'system' coordinate, i.e. the reaction coordinate. Both the 'frozen' reference geometry for the bath [section $2(a)$ ] and a flexible reference geometry [section $2(c)]$ were used to test the sensitivity of the results to how well the bath is modelled.

Table 2 gives the tunnelling splitting for the ground vibrational state of this doublewell potential, as calculated by the basis-set method described in section 3 . Results in table 2 are given for the principal isotope, and also for the deuterated system, each for three different choices of the reference geometry of the bath: 'transition state' refers to a frozen reference geometry of the bath which is that of the transition state, 'equilibrium' also refers to a frozen reference geometry (but one that is the average of the two equilibrium geometries) and 'flexible' refers to the variable reference geometry [section $2(c)$ ] that interpolates between the transition state and equilibrium geometries. The 'exact' values given in table 2 were calculated by the method of Carter and Handy, ${ }^{20}$ which is readily applied to any triatomic system. Finally, the values given in parentheses in table 2 are the results obtained from the one-dimensional vibrationally adiabatic approximation, and are thus a measure of how much coupling to the bath effects the reaction coordinate.

From the results in table 2 one sees that both an equilibrium and a flexible reference geometry for the bath do a better job of describing the dynamics of $\mathrm{H}$-atom transfer (i.e. the tunnelling splitting) than does a frozen transition-state reference geometry; the former results are in quite good agreement with the exact values for this model system. One also sees from table 2 that the bath has a very significant effect on the tunnelling dynamics; viz. the values given by the one dimensional vibrationally adiabatic approximation (those in parenthesis) are in error by 50 to $100 \%$. The error for the equilibrium geometry is the smallest, while that for the transition state is the largest. It thus appears that a quadratic expansion of the potential in bath coordinates about a frozen reference geometry can be sufficient if the geometry of the bath does not relax very much during 
the reaction. A flexible reference is necessary, however, if there is a lot of relaxation in the bath. Fortunately, the flexible-bath model of section $2(c)$ is not any more difficult to apply than is the frozen-bath treatment of section $2(a)$.

\section{Concluding Remarks}

The cartesian reaction-path model presented in section 2 provides a much simpler description of the interaction between the 'system' (i.e. the reaction coordinate) and the 'bath' (the remaining degrees of freedom) than does a reaction-path model based on the (curvilinear) minimum energy (i.e. steepest descent) path. As discussed also, it is often a more physically correct picture of the dynamics (as, for example, in the case of $\mathrm{H}$-atom transfer, where the minimum-energy reaction path is very sharply curved). It is also gratifying that the kind of quantum chemistry calculations that are necessary to apply the model in its $a b$ initio mode are simpler to generate than for the steepest-descent path.

The Hamiltonian that results from this cartesian reaction-path model has the generic form of a cartesian 'system' linearly coupled to a harmonic 'bath'. The most powerful way for treating the dynamics of such a system is Feynman path integral methodology, but it is encouraging to see that the simpler basis-set method summarized in section 3 does quite a good job of describing how coupling to the bath affects the $\mathrm{H}$-atom transfer dynamics. We thus believe that the overall approach described herein will be useful for treating a variety of polyatomic reactive processes.

We gratefully acknowledge support from the National Science Foundation under grant CHE84-16345.

\section{Appendix A}

Here we show briefly why the reaction coordinate always approaches a stationary point from the lowest-frequency mode.

Consider a potential $V=V_{0}+\frac{1}{2} \sum_{i} \omega_{i}^{2} x_{i}^{2}$. For the path of steepest descent

$$
\frac{\partial a}{\partial s} \equiv-\left(\frac{\partial V}{\partial x}\right)_{x=a} /\left|\frac{\partial V}{\partial x}\right|_{x=a}
$$

where $a$ contains the cartesian coordinates of the reaction path as a function of the reaction coordinate $s$. This implies that

$$
\begin{gathered}
\frac{\partial a_{i}}{\partial s} / \frac{\partial a_{j}}{\partial s}=\left(\frac{\partial V}{\partial x_{i}}\right)_{x_{i}=a_{i}} /\left(\frac{\partial V}{\partial x_{j}}\right)_{x_{j}=a_{j}}=\frac{\omega_{i}^{2} a_{i}}{\omega_{j}^{2} a_{j}} \\
\int \frac{1}{a_{i}} \frac{\partial a_{i}}{\partial s} \mathrm{~d} s=\int\left(\frac{\omega_{i}}{\omega_{j}}\right)^{2} \frac{1}{a_{j}} \frac{\partial a_{j}}{\partial s} \mathrm{~d} s
\end{gathered}
$$

and

$$
\ln a_{i}=\left(\frac{\omega_{i}}{\omega_{j}}\right)^{2} \ln a_{j}
$$

Therefore

$$
a_{i}=a_{j}^{\left(\omega_{i} / \omega_{j}\right)^{2}} \rightarrow \text { along the path. }
$$

Examining two possible cases, $(a) \omega_{i}>\omega_{j}$ and $(b) \omega_{i}<\omega_{j}$, one can see that the path of steepest descent approaches the stationary point from the mode of lowest frequency (a) $a_{j}$ and (b) $a_{i}$. 


\section{References}

1 (a) W. H. Miller, N. C. Handy and J. E. Adams, J. Chem. Phys., 1980, 72, 99; (b) W. H. Miller, in Potential Energy Surfaces and Dynamics Calculations, ed. D. G. Truhlar (Plenum Press, New York, 1981), p. 265; (c) C. J. Cerjan, S-H. Shi and W. H. Miller, J. Phys. Chem., 1982, 86, 2244; (d) S. K. Gray, W. H. Miller, Y. Yamaguchi and H. F. Schaefer, J. Chem. Phys., 1980, 73, 2733; (e) S. K. Gray, W. H. Miller, Y. Yamaguchi and H. F. Schaefer, J. Am. Chem. Soc., 1981, 103, 1900; $(f)$ Y. Osamura, H. F. Schaefer, S. K. Gray and W. H. Miller, J. Am. Chem. Soc., 1981, 103, 1094; (g) B. A. Waite, S. K. Gray and W. H. Miller, J. Chem. Phys., 1983, 78, 259; (h) W. H. Miller, J. Phys. Chem., 1983, 87, 3811; (i) T. Carrington Jr, L. M. Hubbard, H. F. Schaefer and W. H. Miller, J. Chem. Phys., 1984, 80, 4347; $(j)$ T. Carrington Jr and W. H. Miller, J. Chem. Phys., 1984, 81, 3573; $(k)$ W. H. Miller, in The Theory of Chemical Reaction Dynamics, ed. D. C. Clary (D. Reidel, Dordrecht, 1986), p. 27; (l) T. Carrington Jr and W. H. Miller, J. Chem. Phys., 1986, 84, 4364.

2 For early work on reaction paths and reaction coordinates, see $(a) \mathrm{S}$. Glasstone, K. J. Laidler and $\mathrm{H}$. Eyring, The Theory of Rate Processes (McGraw-Hill, New York, 1941); (b) R. A. Marcus, J. Chem. Phys., 1966, 45, 4493; 4500; 1968, 49, 2610; (c) G. L. Hofacker, Z. Naturforsch., Teil A, 1963, 18, 607; (d) S. F. Fischer, G. L. Hofacker and R. Seiler, J. Chem. Phys., 1969, 51, 3941; (e) K. Fukui, J. Phys. Chem., 1970, 74, 4161.

3 Some of the recent papers by other workers on reaction-path models are: (a) S. F. Fischer and M. A. Ratner, J. Chem. Phys., 1972, 57, 2769; (b) P. Russegger and J. Brickman, J. Chem. Phys., 1975, 62, 1086; 1977, 60, 1; (c) M. V. Basilevskii, Chem. Phys., 1977, 24, 81; 1982, 67, 337; M. V. Basilevskii and A. G. Shamov, Chem. Phys., 1981, 60, 349; (d) K. Fukui, S. Kato and H. Fujimoto, J. Am. Chem. Soc., 1975, 97, 1; K. Yamashita, T. Yamabe and K. Fukui, Chem. Phys. Lett., 1981, 84, 123; A. K. Fukui, Acc. Chem. Res., 1981, 14, 363; (e) K. Ishida, K. Morokuma and A. Komornicki, J. Chem. Phys., 1977, 66, 2153; $(f)$ A. Nauts and X. Chapuisat, Chem. Phys. Lett., 1982, 85, 212; X. Chapuisat, A. Nauts and G. Durrand, Chem. Phys., 1981, 56, 91; (g) J. Pancir, Collect. Czech. Commun., 1975, 40, 1112; 1977, 42, 16; (h) G. A. Natanson, Mol. Phys., 1982, 46, 481.

4 N. Makri and W. H. Miller, J. Chem. Phys., 1987, 86, 1451.

5 R. Jaquet and W. H. Miller, J. Phys. Chem., 1985, 89, 2139.

6 See, for example, E. B. Wilson Jr, J. C. Decius and P. C. Cross, Molecular Vibrations (McGraw-Hill, New York, 1955), pp. 273-279.

7 See, for example, (a) J. Manz and J. Römelt, Chem. Phys. Lett., 1981, 81, 179; (b) J. A. Kaye and A. Kuppermann, Chem. Phys. Lett., 1981, 77, 573; 1981, 78, 546; (c) V. K. Babamov and R. A. Marcus, J. Chem. Phys., 1981, 74, 1790; (d) C. Hiller, J. Manz, W. H. Miller and J. Römelt, J. Chem. Phys., 1983, 78, 3850; (e) B. C. Garrett, D. G. Truhlar, A. F. Wagner and T. H. Dunning Jr, J. Chem. Phys., $1973,45,120$.

8 R. P. Feynman and A. R. Hibbs, Quantum Mechanics and Path Integrals (McGraw-Hill, New York, 1965).

9 (a) W. H. Miller, S. D. Schwartz and J. W. Tromp, J. Chem. Phys., 1983, 79, 4889; (b) Y. Yamashita and W. H. Miller, J. Chem. Phys., 1985, 82, 5475.

10 (a) A. O. Caldeira and A. J. Leggett, Ann. Phys., 1983, 149, 374; (b) S. Chakravarty and A. J. Leggett, Phys. Rev. Lett., 1984, 52, 5; (c) H. Grabert and U. Weiss, Phys. Rev. Lett., 1985, 54, 1605; (d) A. T. Dorsey, M. P. A. Fisher and M. S. Wartak, Phys. Rev. A, 1986, 33, 1117.

11 (a) D. Thirumalai and B. J. Berne, J. Chem. Phys., 1983, 79, 5029; (b) D. Thirumalai, E. J. Bruskin and B. J. Berne, J. Chem. Phys., 1983, 79, 5063; (c) D. Thirumalai and B. J. Berne, J. Chem. Phys., 1984, 81, 2512.

12 (a) J. D. Doll, J. Chem. Phys., 1984, 81, 3536; (b) D. L. Freeman, R. D. Coalson and J. D. Doll, J. Stat. Phys., 1986, 43, 931.

13 (a) E. C. Behrmann, G. A. Jongeward and P. G. Wolynes, J. Chem. Phys., 1983, 79, 6277; (b) R. W. Hall and P. G. Wolynes, J. Stat. Phys., 1986, 43, 935.

14 (a) D. Chandler and P. Wolynes, J. Chem. Phys., 1981, 74, 4078; (b) M. Sprik, M. L. Klein and D. Chandler, J. Chem. Phys., 1985, 83, 3042; (c) M. Sprik, R. W. Impey and M. L. Klein, J. Chem. Phys., 1985, 83, 5802; (d) C. D. Jonah, C. Romeo and A. Rahman, Chem. Phys. Lett., 1986, 123, 209; (e) P. J. Rossky, J. Schnitker and R. A. Kuharski, J. Stat. Phys., 1986, 43, 949; $(f)$ A. Wallquist, D. Thirumalai and B. J. Berne, J. Chem. Phys., 1986, 85, 1583.

15 I. P. Hamilton and J. C. Light, J. Chem. Phys., 1986, 84, 306.

16 (a) D. Gruner and P. Brumer, Chem. Phys. Lett., 1987, 138, 310; (b) M. Kupka and P. H. Cribb, J. Chem. Phys., 1986, 85, 1303; (c) F. T. Chau, J. Mol. Spectrosc., 1984, 103, 66 and references therein.

17 R. Janoschek, E. G. Weidemann and G. Zundel, J. Chem. Soc., Faraday Trans. 2, 1973, 69, 505.

18 (a) W. F. Rowe, R. W. Duerst and E. B. Wilson, J. Am. Chem. Soc., 1976, 98, 4021; (b) S. L. Baughcum, R. W. Duerst, W. F. Rowe, A. Smith and E. B. Wilson, J. Am. Chem. Soc., 1981, 103, 6296; (c) Z. Smith, E. B. Wilson and R. W. Duerst, Spectrochim. Acta, Part A, 1983, 39, 1117; (d) S. L. Baughcum, Z. Smith, E. B. Wilson and R. W. Duerst, J. Am. Chem. Soc., 1984, 106, 2260; (e) P. Turner, S. L. Baughcum, S. L. Coy and Z. Smith, J. Am. Chem. Soc., 1984, 106, 2265. 
19 J. Bicerano, H. F. Schaefer and W. H. Miller, J. Am. Chem. Soc, 1983, 105, 2550; M. J. Frisch, A. C. Scheiner and H. F. Schaefer, J. Chem. Phys., 1985, 82, 4194.

20 S. Carter and N. C. Handy, Mol. Phys., 1982, 47, 1445; Mol. Phys., 1986, 57, 175.

Paper 7/1587; Received 27th August, 1987 